\title{
Perfil de ácidos graxos da carne de ovelhas de descarte de dois grupos genéticos submetidas a dois sistemas de manejo
}

\author{
Fatty acids profile in meat from culling ewes of two breed submitted to two managing systems
}

\author{
Luis Fernando Vilani de Pelegrini ${ }^{\mathrm{I}}$ Cleber Cassol Pires ${ }^{\mathrm{II}}$ Gilberto Vilmar Kozloski ${ }^{\mathrm{II}}$ \\ Nelcindo Nascimento Terra ${ }^{\text {III }}$ Sueli Regina Baggio ${ }^{\text {IV }}$ Paulo Cezar Bastianello CampagnolV \\ Diego Barcelos Galvani ${ }^{\mathrm{VI}}$ Ricardo Monteiro Chequim ${ }^{\mathrm{VII}}$
}

\section{RESUMO}

O experimento teve como objetivo avaliar se a raça e/ou o sistema de criação afetam o perfil de ácidos graxos da carne de ovelhas de descarte. Foram utilizadas 20 ovelhas, sendo 10 da raça Ideal e 10 da raça Texel, aleatoriamente distribuídas por grupo genético, em dois sistemas de manejo: confinadas e recebendo dieta à base de silagem de sorgo $e$ concentrado ou mantidas em uma pastagem cultivada de clima temperado. Os principais ácidos graxos presentes no músculo Longissimus das ovelhas em todos os tratamentos foram o oléico (C18:1), o palmítico (C16:0) e o esteárico (C18:0). O grupo genético não afetou o perfil de ácidos graxos encontrados nesse músculo. No entanto, os teores de ácidos graxos do tipo ù 3 foram mais altos e a relação ù 6/ù 3 foi mais baixa nas ovelhas mantidas a pasto. Com base no perfil de ácidos graxos, as ovelhas terminadas em pastagem cultivada proporcionaram uma carne mais saudável para o consumo humano que as ovelhas terminadas em confinamento.

Palavras-chave: ácido linoléico conjugado, ácidos graxos saturados, ácidos graxos insaturados, confinamento, pastagem.

\section{ABSTRACT}

The objective of this experiment was to evaluate if the fatty acids profile in meat is affected by sheep breed or managing system. Twenty sheep, being 10 of the Ideal and 10 of the Texel breed, were randomly distributed according to its genetic group in two managing systems: feedlot and receiving sorghum silage and concentrate-based diet or grazing on a temperate pasture. The major fatty acids present in Longissimus muscle of sheep in all treatments were the oleic (C18:1), palmitoleic (C16:0) and stearic (C18:0). The genetic group did not affect the fatty acids profile of this muscle. However, the content of ù 3 fatty acids was higher $(P<.05)$ and the ù $6 / \mathrm{ù} 3$ ratio was lower $(P<.05)$ in meat of sheep grazing the temperate pasture. Based on fatty acids profile, the sheep finished on temperate pasture provided a healthier meat for the human consumption than sheep managed in feedlot.

Key words: conjugated linoleic acid, saturated fatty acids, insaturated fatty acids, feedlot, pasture.

\section{INTRODUÇÃO}

A carne ovina, assim como a dos ruminantes em geral, é rica em ácidos graxos saturados e monoinsaturados, com pequenas quantidades de poliinsaturados (SINCLAIR et al., 1982). O consumo excessivo desse tipo de gordura tem sido associado a doenças cardiovasculares e, em função disso, o consumo de carnes com esta característica tem sido indesejada. No entanto, nos ruminantes, parte significativa dos ácidos graxos insaturados são biohidrogenados no rúmen antes de serem absorvidos. As bactérias responsáveis pela biohidrogenação podem ser divididas em dois grupos. O primeiro grupo é responsável pela biohidrogenação do ácido linoléico

IDepartamento de Medicina Veterinária Preventiva, Universidade Federal de Santa Maria (UFSM), 97105-900, Santa Maria, RS, Brasil. E-mail: pelegrini@smail.ufsm.br. Autor para correspondência.

IIDepartamento de Zootecnia, UFSM, Santa Maria, RS, Brasil.

IIIDepartamento de Tecnologia e Ciência dos Alimentos, UFSM, Santa Maria, RS, Brasil.

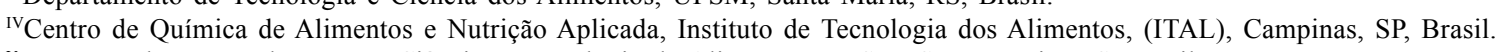

vPrograma de Pós-graduação em Ciência e Tecnologia de Alimentos, UFSM, Santa Maria, RS, Brasil.

VIPorgrama de Pós-graduação em Zootecnia, UFSM, Santa Maria, RS, Brasil.

${ }^{\mathrm{VII}}$ Curso de Medicina Veterinária, UFSM, Santa Maria, RS, Brasil. 
(C18:2ù 6) e do ácido linolênico (C18:3ù 3) a ácido transvacênico (C18:1t11), com pequenas quantidades de outros isômeros. Esse grupo parece ser incapaz de biohidrogenar o ácido graxo oléico (C18:1c9) a ácido esteárico (C18:0). As bactérias do segundo grupo, de outra forma, são capazes de biohidrogenar uma grande extensão de cis e trans C18:1 a C18:0 (DEMEYER \& DOREAU, 1999). Alguns intermediários ao longo da biohidrogenação, como o ácido linoléico conjugado (CLA, C18:2c9t11), são absorvidos pelo animal e são depositados na gordura ou excretados no leite. Tem sido identificado que este ácido graxo tem efeitos anticancerígenos e benéficos à saúde cardiovascular (TAPIERO et al., 2002).

Vários fatores podem afetar o processo de biohidrogenação e a composição dos ácidos graxos depositados na carne dos ruminantes. Dentre eles, destaca-se a composição da dieta, a raça e o sistema de manejo (ENSER et al., 1998, MONTEIRO, 1998; SÃNUDO et al., 2000; WOOD et al., 2004; DEMIREL et al., 2006). No entanto, a maior parte desses estudos avaliou a qualidade da carne de animais jovens. As ovelhas de descarte também podem contribuir para ingresso de receita no sistema de produção e, sendo assim, conhecer os fatores que afetam a qualidade da sua carne torna-se relevante.

Dessa forma, este trabalho teve como objetivo determinar o perfil de ácidos graxos de ovelhas de descarte de dois grupos genéticos terminadas em confinamento ou em pastagem cultivada de aveiaazevém.

\section{MATERIAL E MÉTODOS}

Foram utilizadas 20 ovelhas com a mesma idade (boca cheia com desgaste visível das pinças) e condição corporal de 2,5 (em uma escala onde $1=$ muito magro e 5= muito gordo), sendo 10 da raça Ideal e 10 da raça Texel. Essas ovelhas foram aleatoriamente distribuídas por grupo genético, num delineamento inteiramente casualizado, nos seguintes sistemas de manejo: confinadas e recebendo dieta à base de silagem de sorgo e concentrado ou mantidas em pastagem cultivada de aveia preta (Avena strigosa Schreb.) e azevém (Lolium multiflorum Lam.).

A pastagem foi implantada pelo sistema de preparo mínimo, utilizando-se uma mistura de aveia preta (Avena strigosa Schreb.) e azevém (Lolium multiflorum Lam.), cuja densidade de sementes foi de 70 e $25 \mathrm{~kg} \mathrm{ha}^{-1}$, respectivamente. A adubação de base foi de $200 \mathrm{~kg} \mathrm{ha}^{-1}$ de adubo NPK na fórmula 5-20-20, sendo distribuídos, ainda, $90 \mathrm{~kg} \mathrm{ha}^{-1} \mathrm{de} \mathrm{N}$, na forma de uréia, em três aplicações. A área total da pastagem foi subdividida em dois piquetes com área média de 0,40 ha, sendo alocadas, em cada um destes, cinco ovelhasteste e um número variável de reguladoras. A massa e a oferta de forragem pretendidas durante o período experimental foram de, respectivamente, $1.043 \mathrm{~kg} \mathrm{ha}^{-1}$ de matéria seca (MS) e $10 \mathrm{~kg}$ MS $100 \mathrm{~kg}$ de peso vivo ${ }^{-1}$.

A dieta fornecida aos animais confinados foi formulada para conter $13 \%$ de proteína bruta (PB) e $75 \%$ de nutrientes digestíveis totais (NDT) na MS (NRC, 1985). Foi constituída de 59,5\% de silagem de sorgo (Sorghum bicolor (L.) Moench), 25,5\% de grão de milho (Zea mays L.) triturado, 13,8\% de farelo de soja (Glycine max L.), 0,6\% de sal comum, 0,54\% de calcário calcítico e $0,10 \%$ de fosfato bicálcico. Os animais foram alimentados ad libitum duas vezes ao dia, em baias individuais, às $8 \mathrm{~h}$ às $17 \mathrm{~h}$, em quantidades de forma a terem sobras de $10 \%$ do oferecido.

Os animais foram mantidos no confinamento e na pastagem até atingirem a condição corporal de 3,5, quando então foram abatidos após jejum de sólidos por 14 horas. As carcaças foram refrigeradas durante 24 horas em câmara fria com temperatura entre 0 e $4^{\circ} \mathrm{C}$. Logo após, foi realizada a classificação das carcaças, cortes e desossa. O músculo Longissimus foi retirado seccionando-o entre a $12^{\mathrm{a}}$ e a $13^{\mathrm{a}}$ costela e congelado para posterior análise do perfil de ácidos graxos.

A extração dos lipídios da amostra do Longissimus foi feita segundo a metodologia de BLIGH \& DYER (1959). Dez gramas de amostra foram triturados, colocados em erlemeyer com $10 \mathrm{~mL}$ de clorofórmio e $20 \mathrm{~mL}$ de metanol e agitados o por 5 minutos. A seguir, foram acrescidos $10 \mathrm{~mL}$ de metanol, novamente agitado o conteúdo por 5 minutos, sendo depois transferido para um funil de separação. A parte superior da solução consistiu de metanol, água e extratos não-lipídicos, tendo sido descartada. A parte inferior da solução, clorofórmio e lipídios, foi extraída para determinação de ácidos graxos. Para a análise dos ácidos graxos, uma alíquota do extrato lipídico, contendo aproximadamente $200 \mathrm{mg}$ de lipídios, foi secada em evaporador rotatório e transmetilada de acordo com o método de HARTMAN \& LAGO (1973), usando-se solução de cloreto de amônia e ácido sulfúrico em metanol como agente esterificante. Os ácidos graxos foram determinados por cromatografia gasosa, utilizando-se um cromatógrafo marca Varian, modelo 3900, com coluna capilar CP-SIL 88 (100m X $0,25 \mathrm{~mm}$ d.i., 0,20um de filme). As condições cromatográficas foram: temperatura inicial: $120^{\circ} \mathrm{C} 5 \mathrm{~min}^{-1}$, elevando-se para $235^{\circ} \mathrm{C}$ numa escala de $3^{\circ} \mathrm{C} \mathrm{min}{ }^{-1}$, permanecendo nesta temperatura por 20 minutos; gás de arraste: hidrogênio numa vazão de $1 \mathrm{~mL} \mathrm{~min}^{-1}$; gás make-up: nitrogênio a $30 \mathrm{~mL} \mathrm{~min}^{-1}$; temperatura do injetor: $270^{\circ} \mathrm{C}$; temperatura do detector: $300^{\circ} \mathrm{C}$; e volume de injeção: $1 u L$. 
A identificação dos ácidos graxos foi realizada através da comparação do tempo de retenção dos ácidos graxos das amostras com o de padrões conhecidos. O teor de cada ácido graxo na amostra do Longissimus foi calculada como segue:

$$
\mathrm{AGi}=\frac{\mathrm{A} \times \mathrm{L} \times \mathrm{F}}{100}
$$

em que:

$\mathrm{AGi}=$ teor do ácido graxo na amostra $\left(\mathrm{g} 100 \mathrm{~g}^{-1}\right)$;

$\mathrm{A}=$ porcentagem de área de cada um dos picos obtidos nos cromatogramas;

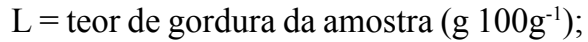

$\mathrm{F}=0,910$, fator que corrige o teor de gordura para componentes lipídicos que não são ácidos graxos (HOLLAND et al., 1994).

Os dados foram submetidos à análise de variância, cujo modelo incluiu os efeitos de genótipo, sistema alimentar, interação genótipo $\times$ sistema alimentar e o erro experimental, sendo utilizado o teste $\mathrm{F}$ para verificar a significância dos efeitos testados. As análises foram realizadas com auxílio do programa estatístico SAS (1997).

\section{RESULTADOS E DISCUSSÃO}

O teor de gordura total não foi influenciado pela raça nem pelo sistema de manejo (Tabela 1) e representou, em média, $3,59 \%$ do músculo Longissimus. O teor de gordura das ovelhas foi similar ao de cordeiros de diferentes raças submetidos a diferentes sistemas de manejo (MONTEIRO et al., 1995; VELASCO et al., 2004). No estudo de MONTEIRO et al. (1995), no entanto, o teor de gordura do Longissimus foi mais alto nos cordeiros das raças Corriedale e Romney Marsh, se comparados aos das cruzas Texel $\times$ Corriedale e Ideal $\times$ Merino ( média de 4,01 vs, 3,69, respectivamente).

A composição individual dos ácidos graxos do músculo Longissimus nas duas raças e sistemas de manejo estudados é apresentada na tabela 1, sendo os mesmos ácidos agrupados com base no tipo e grau de saturação, na tabela 2. Não foi observado efeito do grupo genético no perfil de ácidos graxos. Os principais ácidos graxos presentes no Longissimus das ovelhas foram o oléico (C18:1 $\omega 9)$, o palmítico (C16:0) e o esteárico (C18:0), os quais representaram em torno de $75 \%$ da gordura total em todos os tratamentos. O coeficiente de variação da medida dos teores dos ácidos graxos individuais foi alto, indicando que um número maior de repetições deve ser utilizado em estudos desta natureza.

Os ácidos graxos de particular interesse, devido aos seus efeitos positivos à saúde humana, são principalmente o linolênico (C18:3ù 3$)$ e o linoléico (C18:2ù 6), os quais são considerados essenciais, além dos ácidos graxos com insaturação no carbono ù 3 e o
CLA. O teor deste último não foi influenciado, mas o teor da forma trans do linoléico (C18:2ù 6T), assim como os teores de C18:3ù 3 e C20:5ù 3, foram mais altos nos animais mantidos em pastagem. Vários outros estudos que compararam a qualidade da gordura da carne de cordeiros confinados ou em pastagem também observaram tal tendência (ROWE et al., 1999; FISHER et al., 2000; SÃNUDO et al., 2000; DEMIREL et al., 2006). $\mathrm{O}$ teor dos ácidos graxos da dieta no presente estudo não foi medido, mas, normalmente, a forragem verde contém relativamente maiores teores de C18:3ù 3 (DÍAZ et al., 2002) e menores de C18:2ù 6 que grãos e/ou concentrados (ROWE et al., 1999). Esses resultados indicam que a biohidrogenação dos ácidos graxos no rúmen é incompleta e que a extensão desse processo varia com o tipo de dieta. Houve interação dos efeitos raça e sistema de manejo na concentração de C20:5ù 3 . No entanto, não foi encontrada na literatura nenhuma referência que indique que esse ácido graxo em particular tenha algum efeito específico em relação à saúde humana. Os ácidos graxos poliinsaturados localizados nas membranas celulares são precursores de diferentes eicosanóides (prostaglandinas, tromboxanos e leucotrienos), que atuam como mensageiros da célula e reguladores metabólicos, cujas funções específicas são particularmente de grande interesse no estudo de doenças cardiovasculares. Além desse fator, os benefícios de seu uso estão associados à sua performance na manutenção da integridade da membrana celular e à sua capacidade em diminuir a quantidade de lipídios séricos (EDER, 1995).

A proporção de ácidos graxos ù $6 /$ ù 3 também tem sido utilizada como um critério para avaliar a qualidade da gordura, a qual deveria ser inferior a 4 (DEPARTMENT OF HEALTH, UK, 1994). No presente trabalho, esta relação não foi influenciada pela raça, mas foi marcadamente inferior nas ovelhas terminadas em pastagem. Da mesma forma, estes animais tiveram maior conteúdo de ácidos graxos poliinsaturados e de ácidos graxos desejáveis totais que os terminados em confinamento. Este efeito positivo da pastagem sobre o perfil de ácidos graxos também foi observado em cordeiros por ROWE et al. (1999) e DÍAZ et al. (2002). O teor médio de ácidos graxos desejáveis totais verificado neste estudo (em torno de $71 \%$ da gordura) é similar ao normalmente presente na carne de ovinos (RHEE, 1992; BANSKALIEVA et al., 2000; MADRUGA et al., 2005).

A proporção de ácidos graxos poliinsaturados/saturados das ovelhas no presente estudo, no entanto, foi em média de 0,10 , inferior a 0,45 , valor que é recomendado como mínimo ideal na dieta humana(WOOD \& ENSER, 1997).

\section{CONCLUSÃO}

Nas condições do presente estudo, a gordura da carne de ovelhas de descarte, 
Tabela 1 - Teor de gordura e de ácidos graxos individuais $\left(\mathrm{g} 100 \mathrm{~g}^{-1}\right)$ no músculo Longissimus de ovelhas de descarte terminadas em confinamento ou em pastagem cultivada de inverno.

\begin{tabular}{|c|c|c|c|c|c|c|c|c|c|}
\hline & \multicolumn{2}{|c|}{ Raça } & \multicolumn{2}{|c|}{ Sistema de manejo } & \multirow{2}{*}{ Média } & \multirow{2}{*}{$\mathrm{CV}^{\mathrm{b}}(\%)$} & \multirow{2}{*}{ Raça } & \multirow{2}{*}{$\begin{array}{l}\text { Sistema de } \\
\text { manejo }\end{array}$} & \multirow{2}{*}{ Interação ${ }^{c}$} \\
\hline & Ideal & Texel & Confin. & Pastag. & & & & & \\
\hline Gordura $^{\mathrm{d}}$ & 3,93 & 3,25 & 3,66 & 3,52 & 3,59 & 45,15 & ns & ns & ns \\
\hline $\mathrm{C} 10: 0$ & 0,0038 & 0,0025 & 0,0036 & 0,0028 & 0,0032 & 47,37 & ns & ns & ns \\
\hline C14:0 & 0,0749 & 0,0560 & 0,0684 & 0,0624 & 0,0654 & 39,45 & ns & ns & ns \\
\hline $\mathrm{C} 15: 0$ & 0,0143 & 0,0130 & 0,0128 & 0,0145 & 0,0135 & 40,52 & ns & ns & ns \\
\hline C16:0 & 0,8672 & 0,7004 & 0,8283 & 0,7393 & 0,7838 & 43,01 & ns & ns & ns \\
\hline $\mathrm{C} 17: 0$ & 0,0356 & 0,0303 & 0,0320 & 0,0338 & 0,0329 & 46,87 & ns & ns & ns \\
\hline C18:0 & 0,7202 & 0,5768 & 0,6408 & 0,6562 & 0,6485 & 42,94 & ns & ns & ns \\
\hline $\mathrm{C} 20: 0$ & 0,0036 & 0,0029 & 0,0033 & 0,0032 & 0,0033 & 44,21 & ns & ns & ns \\
\hline $\mathrm{C} 22: 0$ & 0,0055 & 0,0045 & 0,0035 & 0,0065 & 0,0050 & 46,21 & ns & $* *$ & ns \\
\hline $\mathrm{C} 14: 1 \varpi 5$ & 0,0125 & 0,0104 & 0,0102 & 0,0127 & 0,0114 & 56,01 & ns & ns & ns \\
\hline $\mathrm{C} 16: 1 \varpi 7$ & 0,0599 & 0,0512 & 0,0597 & 0,0514 & 0,0556 & 48,56 & ns & ns & ns \\
\hline C17:1 & 0,0191 & 0,0159 & 0,0168 & 0,0182 & 0,0175 & 45,02 & ns & ns & ns \\
\hline C18:1 ఐ9 & 1,3614 & 1,1740 & 1,3330 & 1,2023 & 1,2677 & 45,56 & ns & ns & ns \\
\hline C18:1 ఐ9t & 0,1335 & 0,0977 & 0,0881 & 0,1431 & 0,1156 & 51,12 & ns & ns & ns \\
\hline C18:2 ఐ6 & 0,0752 & 0,0514 & 0,0673 & 0,0593 & 0,0633 & 44,88 & ns & ns & ns \\
\hline $\mathrm{C} 18: 2 \varpi 6 \mathrm{t}$ & 0,0283 & 0,0206 & 0,0160 & 0,0330 & 0,0245 & 48,54 & ns & $* *$ & ns \\
\hline $\mathrm{C} 18: 2 \mathrm{c} 9 \mathrm{t} 11^{\mathrm{a}}$ & 0,0344 & 0,0257 & 0,0232 & 0,0369 & 0,0301 & 56,68 & ns & ns & ns \\
\hline C18:3 & 0,0262 & 0,0158 & 0,0144 & 0,0276 & 0,0210 & 55,21 & ns & $*$ & ns \\
\hline $\mathrm{C} 20: 4 \varpi 6$ & 0,0175 & 0,0126 & 0,0152 & 0,0149 & 0,0150 & 37,70 & ns & ns & ns \\
\hline $\mathrm{C} 20: 5 \varpi 3$ & 0,0062 & 0,0044 & 0,0036 & 0,0070 & 0,0053 & 47,64 & ns & $* *$ & $* *$ \\
\hline $\mathrm{C} 22: 6 \varpi 3$ & 0,0044 & 0,0057 & 0,0055 & 0,0046 & 0,0051 & 61,52 & ns & ns & ns \\
\hline
\end{tabular}

á́cido linoléico conjugado (CLA).

${ }^{\mathrm{b} C o e f i c i e n t e ~ d e ~ v a r i a c ̧ a ̃ o . ~}$

${ }^{\mathrm{c}} \mathrm{Raça} \times$ sistema de manejo.

${ }^{\mathrm{d}} \mathrm{C} 10: 0=$ ácido cáprico; $\mathrm{C} 14: 0=$ ácido mirístico; $\mathrm{C} 15: 0=$ ácido pentadecanóico; $\mathrm{C} 16: 0=$ ácido palmítico; $\mathrm{C} 17: 0=$ ácido heptadecanóico; C18:0 = ácido esteárico; C20:0 = ácido araquídico; C22:0 = ácido behênico; C14:1 55 ácido miristoléico; C16:1 $77=$ ácido palmitoléico;

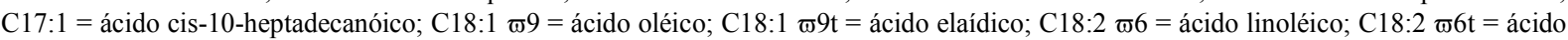

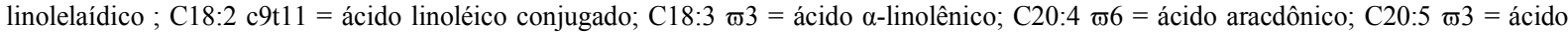
eicosapentaenóico; C22:6 $\varpi 3$ = ácido docosahexaenóico.

independentemente da raça, apresentou um perfil de ácidos graxos de cadeia longa mais favorável à saúde humana se terminadas em pastagem temperada do que em confinamento e recebendo silagem e concentrado.

\section{AGRADECIMENTOS E APRESENTAÇÃO}

Ao Conselho Nacional de Desenvolvimento Científico e Tecnológico (CNPq), pela bolsa concedida aos pesquisadores Cleber Cassol Pires e Nelcindo Nascimento Terra.

Tabela 2 - Proporção dos diferentes grupos de ácidos graxos do músculo Longissimus de ovelhas de descarte terminadas em confinamento ou em pastagem cultivada de inverno.

\begin{tabular}{|c|c|c|c|c|c|c|c|c|c|}
\hline & \multicolumn{2}{|c|}{ Raça } & \multicolumn{2}{|c|}{ Sistema alimentar } & \multirow{2}{*}{ Média } & \multirow{2}{*}{ CV (\%) } & \multirow{2}{*}{ Raça } & \multirow{2}{*}{$\begin{array}{l}\text { Sistema } \\
\text { alimentar }\end{array}$} & \multirow{2}{*}{$\begin{array}{c}\text { Raça } \times \\
\text { Sistema } \\
\text { alimentar }\end{array}$} \\
\hline & Ideal & Texel & Conf. & Pastag & & & & & \\
\hline Saturados $^{\mathrm{a}}$ & 49,52 & 48,57 & 49,45 & 48,65 & 49,05 & 4,48 & ns & ns & ns \\
\hline Monoinsaturados $^{\mathrm{a}}$ & 45,08 & 46,53 & 46,11 & 45,51 & 45,81 & 4,42 & ns & ns & ns \\
\hline Polinsaturados $^{\mathrm{a}}$ & 5,39 & 4,87 & 4,44 & 5,85 & 5,14 & 11,31 & ns & $* *$ & ns \\
\hline $\mathrm{AGD}^{\mathrm{b}}$ & 71,15 & 71,72 & 70,32 & 72,56 & 71,43 & 2,19 & ns & $* *$ & ns \\
\hline AGP:AGS ${ }^{\mathrm{c}}$ & 0,11 & 0,10 & 0,09 & 0,12 & 0,11 & 12,56 & ns & $* *$ & ns \\
\hline$\omega 6: \omega 3^{\mathrm{d}}$ & 3,96 & 3,41 & 4,63 & 2,74 & 3,68 & 23,09 & $\mathrm{~ns}$ & $* *$ & ns \\
\hline
\end{tabular}

a $\%$ dos ácidos graxos totais.

b Ácidos graxos desejáveis, representados pela soma das proporções de C 18:0 + AGP (poliinsaturados) + AGMI (monoinsaturados).

${ }^{\mathrm{c}}$ Relação entre o teor de ácidos graxos poliinsaturados (AGP) e os saturados (AGS).

${ }^{\mathrm{d}}$ Relação entre o teor de ácidos graxos poliinsaturados ômega 6 e os ômega 3 . 
Parte da Tese de Doutorado apresentada pelo primeiro autor à Universidade Federal de Santa Maria (UFSM), Santa Maria, RS, Brasil.

\section{REFERÊNCIAS}

BANSKALIEVA, V. et al. Fatty acid composition of goat muscles and fat depots: a review. Small Ruminant Research, v.37, n.3, p.255-268, 2000.

BLIGH, E.G.; DYER, W.J. Canadian Journal of Biochemistry and Physiology, v.37, p.911-917, 1959.

DEMEYER, D.; DOREAU, M. Targets and procedures for altering ruminant meat and milk lipids. Proceedings of the Nutrition Society, v.58, n.3, p.593-607, 1999

DEMIREL, G. et al. Fatty acids of lamb meat from two breeds fed different forage: concentrate ratio. Meat Science, v.72, n.2, p. 229-235, 2006.

DEPARTMENT OF HEALTH. Nutritional aspects of cardiovascular disease: report of the cardiovascular review group. London: HMSO, 1994. (Report on health and social subjects; 46).

DÍAZ, M.T. et al. Use of concentrate or pasture for fattening lambs and its effect on carcass and meat quality. Small Ruminant research, v.43, n.3, p.257-268, 2002.

EDER, K. Gas cromatographic analysis of fatty acid methyl esters. Journal of Chromatography, v.671, p.113-131, 1995.

ENSER, M. et al. Fatty acid content and composition of UK beef and lamb muscle in relation to production system and implications for human nutrition. Meat Science, v.49, n.3, p.329-341, 1998 .

FISCHER, A.V. et al. Fatty acid composition and eating quality of lamb types derived from four diverse breed x production systems. Meat Science, v.55, n.2, p.141-147, 2000.

HARTMAN, L.; LAGO, R.C.A. Rapid preparation of fatty acid methyl esters from lipids. Laboratory Practice, v.22, n. 8, p.475-476, 1973 .

HOLLAND, B. et al. McCance \& Widdowson's the composition of foods. 4.ed. Cambridge: Royal Society of Chemistry and Ministry of Agriculture, Fisheries and Food London, 1994. p.8-9.
MADRUGA, M.S. et al. Qualidade da carne de cordeiros Santa Inês terminados com diferentes dietas. Revista Brasileira de Zootecnia, v.34, n.1, p.309-315, 2005.

MONTEIRO, E.M. Influência do cruzamento Ile de France x Corriedale (F1) nos parâmetros de qualidade da carne de cordeiro. 1998. 99f. Tese (Doutorado em Ciência dos Alimentos) - Universidade de São Paulo.

MONTEIRO, E. et al. Composição física de cinco raças ovinas. In: SIMPOSIO LATINO AMERICANO DE CIENCIAS DOS ALIMENTOS, 1995, Campinas, SP. Anais... Campinas: Faculdade de Engenharia de Alimentos - FEA, 1995. CD-ROM.

NRC - NATIONAL RESEARCH COUNCIL. Nutrient requeriments of sheep. Washington: National Academy of Sciences, 1985. 112p.

RHEE, K.S. Fatty acids in meats and meat products. In: CHOW, C.K. (Ed.). Fatty acids in foods and their health implications. New York: Marcel Dekker, 1992. p.65-93.

ROWE, A. et al. Muscle composition and fatty acid profile in lambs fattened in drylot or pasture. Meat Science, v.51, n.4, p.283-288, 1999.

SAÑUDO, C. et al. Fatty acid composition and sensory characteristics of lamb carcasses from Britain and Spain. Meat Science, v.54, n.4, p.339-346, 2000

SAS - STATISTICAL ANALISYS SYSTEM. User's guide. Versão 6. 4.ed. North Caroline, 1997. 846p.

SINCLAIR, A.J. et al. The analysis of polyunsaturated fatty acid in meat by capillary gas-liquid chromatography. Journal Science Food Agriculoture, v.33, n.8, p.771-776, 1982.

TAPIERO, H. et al. Polynsaturated fatty acids (PUFA) and eicosanoids in human health and pathologies. Biomedicine \& Pharmacotherapy, v.56, n.5, p.215-222, 2002.

VELASCO, S. et al. Effect of different feeds on meat quality and fatty acid composition of lambs fattened at pasture. Meat Science, v.66, n.2, p.457-465, 2004

WOOD, J.D.; ENSER, M. Factors influecing fatty acids in meat and the role of antioxidants in improving meat quality. Brittish Journal of Nutrition, v.78, n.1, p.49-60, 1997

WOOD, J.D. et al. Effects of fatty acids on meat quality: a review. Meat Science, v.66, n.1, p.21-32, 2004. 